Clarke, P. H., Glover, P. \& Mathias, A. P. (1959). J. gen. Microbiol. 20, 156-164.

\title{
The Occurrence of Polyol Derivatives of Cytidine Diphosphate in Micro-organisms
}

\author{
By PATRICIA H. ClaRKE, PHILOMENA GLOVER ANd A. P. MATHIAS \\ Department of Biochemistry, University College London
}

\begin{abstract}
SUMMARY: A survey of cytidine diphosphate compounds in representative species of Gram-positive and Gram-negative bacteria has been made. Cytidine diphosphate glycerol and cytidine diphosphate ribitol, first discovered in Lactobacillus arabinosus 17/5, were also found in $L$. plantarum. Cytidine diphosphate glycerol was found in L. bulgaricus and cytidine diphosphate ribitol in Bacillus subtilis. No correlation between the presence of cytidine nucleotides and the Gram reaction was found.
\end{abstract}

Two derivatives of cytidine diphosphate were found in extracts of heat-killed Lactobacillus arabinosus 17/5 (Baddiley \& Mathias, 1954) and were shown to be cytidine diphosphate glycerol (CDPglycerol; Baddiley, Buchanan, Mathias \& Sanderson, 1956) and cytidine diphosphate ribitol (CDPribitol; Baddiley, Buchanan, Carss \& Mathias, 1956). The $\alpha$-glycerophosphate moiety of CDPglycerol has the L-configuration (Baddiley, Buchanan \& Carss, 1956) in common with the $\alpha$-glycerophosphate isolated from other natural sources (Baer \& Fischer, 1939). By enzymic methods it has been shown that CDPribitol contains a D-ribitol-5-phosphate residue, which probably would be formed by the reduction of D-ribose-5-phosphate. Two other cytidine diphosphate derivatives are known: cytidine diphosphate choline and cytidine diphosphate ethanolamine. These are involved in the incorporation of phosphoryl choline or phosphoryl ethanolamine into $\alpha \beta$-diglycerides with the formation of lecithins or cephalins (Kennedy \& Weiss, 1956). It is not yet known what roles CDPglycerol and CDPribitol play in the metabolism of glycerol and ribitol.

Mitchell \& Moyle $(1951 a, b)$ isolated a glycerophosphate compound, mainly occurring in the cell envelope and small particle fractions of Staphylococcus aureus (Micrococcus pyogenes). No evidence was presented for the structure of this material, but the ease of hydrolysis in alkali fits in well with a linear polymer composed of glycerol residues linked $1: 2$ or $1: 1$ through a phosphodiester group. Mitchell \& Moyle (1954) examined a number of Gram-positive and Gram-negative organisms, and reported the presence of other unidentified polyol phosphates in Gram-positive organisms and suggested that a lipidpolyol phosphate-protein component might be a common structural feature of Gram-positive bacteria. If one of the unidentified polyol phosphates were ribitol phosphate, the cells might contain a polyribitol phosphate, or a material in which either ribitol or glycerol residues were joined through a phosphodiester link. If such compounds did indeed exist in bacteria, then it would be reasonable to suppose that CDPribitol and CDPglycerol were involved in their biosynthesis. Furthermore, if the suggestion of Mitchell \& Moyle (1954) 
were right, one might expect to find CDPribitol and CDPglycerol in extracts of Gram-positive organisms, but not in Gram-negative organisms. Accordingly, a survey was made to determine the distribution of the cytidine diphosphate compounds. The organisms investigated included most of those used by Mitchell $\&$ Moyle (1954) and a number of lactobacilli.

\section{METHODS}

Cultures and growth conditions. Lactobacillus arabinosus $17 / 5$ was obtained from Dr D. E. Hughes, L. plantarum NCIB 8030 from the National Collection of Industrial Bacteria, and the other cultures from the National Collection of Type Cultures (NCTC). Streptococcus lactis H 240 came originally from the collection of Dr P. M. F. Shattock.

Lactobacillus arabinosus $17 / 5$ was grown under the conditions described by Baddiley \& Mathias (1954) in 5 l. batches. L. bulgaricus NCTC 76 and $L$. plantarum NCIB 8030 were subcultured from milk into $100 \mathrm{ml}$. flasks containing $50 \mathrm{ml}$. tomato juice broth (Briggs, 1953) and these were used to inoculate four flasks, each containing $2.5 \mathrm{l}$. of the same medium, which were incubated at $37^{\circ}$ for 2 days.

Escherichia coli NCTC 86, Klebsiella aerogenes NCTC 418 and Bacillus cereus NCTC 945 were grown in $1 \%(w / v)$ glucose broth containing $(\%, w / v)$ : Lab. Lemco, 1; peptone, 1 ; sodium chloride, 0.5 ; glucose, 1 ; made up in distilled water. Each batch consisted of 81 . of medium distributed as 21 . in each of four 5 l. Pyrex bottles, which were aerated by continuous rotation on an electrically-driven mechanical rotator for 18-24 hr. at about $27^{\circ}$.

Streptococcus lactis $\mathrm{H} 240$ and Staphylococcus aureus NCTC 4163 were grown under the same conditions as Escherichia coli with the medium supplemented by the addition of $25 \mathrm{ml}$. of a $10 \%(\mathrm{w} / \mathrm{v})$ yeast extract/l. medium. For the experiment on the effect of penicillin on nucleotide accumulation, Staphylococcus aureus NCTC 4163 was grown for $12 \mathrm{hr}$. in $4 \mathrm{l}$. of medium as above, the cocci were harvested by centrifugation in an MSE refrigerated centrifuge at $1500 \mathrm{~g}$ for $30 \mathrm{~min}$. and resuspended in $2 \mathrm{l}$. of fresh broth containing 10 units penicillin $/ \mathrm{ml}$. and incubated for a further $3 \mathrm{hr}$.

Bacillus subtilis NCTC 3610 was inoculated from a Lemco agar slope culture into 20 tubes, each containing approximately $10 \mathrm{ml}$. of $1 \%(\mathrm{w} / \mathrm{v})$ glucose broth, which were incubated with shaking for $8 \mathrm{hr}$. at $37^{\circ}$. The broth cultures were used to inoculate 40 Roux bottles each containing $100 \mathrm{ml}$. Lemco agar. The Roux bottles were incubated $18 \mathrm{hr}$. at $37^{\circ}$ and the organisms washed off with $c .1$ l. distilled water, filtered through gauze and centrifuged for $15 \mathrm{~min}$. in an MSE Angle 13 centrifuge at $4000 \mathrm{~g}$.

Preparation of the nucleotide extracts from the bacteria. The organisms, grown in liquid culture, were harvested in a Sharples centrifuge, weighed and immediately extracted with aqueous ethanol $(65 \%, \mathrm{v} / \mathrm{v})$, allowing $30 \mathrm{ml} . / 10 \mathrm{~g}$. wet organisms. The mixture was heated rapidly to the boiling point with vigorous stirring, boiled for $5 \mathrm{~min}$., cooled and centrifuged. The precipitate was extracted with a similar volume of boiling aqueous ethanol $(50 \%, \mathrm{v} / \mathrm{v})$. 
The combined extracts were brought to $\mathrm{pH} 5$ with dilute nitric acid and a slight excess of a solution of mercuric acetate in acetic acid added (Caputto, Leloir, Cardini \& Paladini, 1950). After $16 \mathrm{hr}$. at $0^{\circ}$, the precipitate was centrifuged down and washed with a little ethanol $(50 \%, v / v)$. It was suspended in water and a vigorous stream of hydrogen sulphide passed through the suspension to decompose the mercury salts. The mercury sulphide was removed by centrifugation, the supernatant fluid aerated to remove hydrogen sulphide, adjusted to $\mathrm{pH} 8$ with ammonia, and freeze-dried.

Ion-exchange chromatography. A column $(1.5 \times 17.5 \mathrm{~cm}$. $)$ of Dowex-1 (formate-form; 200-400 mesh) was prepared according to the method of Hurlbert, Schmitz, Brumm \& Potter (1954). A solution of the nucleotide ammonium salts $(0 \cdot 2-0.7 \mathrm{~g}$.) in water $(25-50 \mathrm{ml}$.), adjusted to pH 8.5, was added to the column at a rate of $0.3 \mathrm{ml} . / \mathrm{min}$. The column was then washed with water until the absorption of the eluate at $260 \mathrm{~m} \mu$ had fallen to $0 \cdot 1$. Elution was effected by the use of a linear gradient of formic acid. This was obtained by employing two identical cylindrical vessels as the reservoir and the mixing flasks, which were connected by a syphon (Drake, 1955). The mixing flask was joined to the column by a short length of polythene tubing. Approximately $10 \mathrm{ml}$. of liquid were kept above the surface of the resin to prevent disturbance by splashing. The solution in the mixing vessel was stirred 'magnetically'. At the start, the mixing vessel contained $1 \mathrm{l}$. of water and the reservoir $1 \mathrm{l}$. of $5 \mathrm{~N}$-formic acid. The flow was adjusted to $0 \cdot 3-0 \cdot 4 \mathrm{ml} . / \mathrm{min}$., and the eluate collected in $7 \cdot 2 \mathrm{ml}$. fractions. The absorption at $260 \mathrm{~m} \mu$ of each fraction was measured in a $1 \mathrm{~cm}$. cell. To help in the identification of the base, the absorption at $275 \mathrm{~m} \mu$ of the tubes corresponding to the maximum of each peak was also determined. The fractions containing each peak were combined and freeze-dried. At the end of each run, the column was regenerated by treatment with a solution of $6 \mathrm{~N}$-formic acid containing $4 \mathrm{~N}$-ammonium formate.

Paper chromatography of the nucleotide peaks. Each peak-eluate was examined by ascending chromatography on Whatman no. 4 paper, previously washed with $2 \mathrm{~N}$-acetic acid followed by water. The solvents used were: isobutyric acid +ammonium isobutyrate + water (Magasanik et al. 1950); propanol+ ammonia + water (Hanes \& Isherwood, 1949); ethanol + ammonium acetate (Paladini \& Leloir, 1952). Several samples of each eluate were run in each solvent together with the appropriate markers. The papers also had blank tracks. After chromatography and removal of the solvents, the chromatograms were examined under ultraviolet radiation and the absorbing spots marked with pencil. The paper was cut into strips. The u.v.-absorbing spots from one track were cut out, eluted with $3 \mathrm{ml}$. of $0.01 \mathrm{~N}$-hydrochloric acid, and the u.v. absorption spectrum determined, using as a blank the eluate from a spot of similar area and $\mathbf{R}_{F}$ taken from a blank track. The eluates were then adjusted to $\mathrm{pH} 12$ by the addition of $2 \mathrm{~N}$-sodium hydroxide, and the spectrum redetermined. The other tracks were treated with one of the following sprays : the phosphate reagents of Hanes \& Isherwood (1949); the periodate-Schiff reagent (Baddiley, Buchanan, Handschumacher \& Prescott, 1956), and in the 
case of suspected nicotinamide derivatives, with the methylethylketone + ammonia reagent (Kodicek \& Reddi, 1951). In the few cases in which the base could not be definitely identified from the u.v. absorption spectrum, another sample of the band under investigation was eluted from the paper, evaporated to dryness and hydrolysed with perchloric acid $(72 \%, w / v)$ according to the method of Marshak \& Vogel (1951). The hydrolysate was run on paper with the isopropanol + hydrochloric acid system (Wyatt, 1951), and the purine or pyrimidine base identified by comparison of its $\boldsymbol{R}_{F}$ value with that of authentic specimens and from its u.v. absorption spectrum.

The cytidine compounds from peaks $\mathrm{C}$ and $\mathrm{D}$ were readily distinguishable on paper in the isobutyric acid solvent, having $R_{F}$ values of 0.15 and 0.22 , respectively. Both reacted rapidly with the periodate spray. To confirm the structures assigned to them, samples were eluted from paper chromatograms with a few drops of $\mathrm{N}$-hydrochloric acid, and the eluate heated in a sealed tube at $100^{\circ}$ for $2-3 \mathrm{hr}$. After evaporation of the acid, the hydrolysate was separated chromatographically with the propanol+ammonia solvent. The products were detected by examination under u.v. radiation and by spraying with the phosphate and the periodate-Schiff reagents. CDPribitol gave CMP and mainly anhydro-ribitol. CDPglycerol gave CMP and glycerophosphate.

\section{RESUL'TS}

The same column of ion-exchange resin was used throughout these experiments and was standardized by an examination of the nucleotides from heatkilled Lactobacillus arabinosus 17/5, which have already been investigated (Baddiley, Buchanan, Carss, Mathias \& Sanderson, 1956). The elution pattern obtained from L. arabinosus is shown in Fig. 1. The other organisms gave similar patterns, with the exception of peaks $\mathrm{D}$ and $\mathrm{E}$, and there would seem to be no gross differences amongst them in the amounts of DPN, AMP and ADP present.

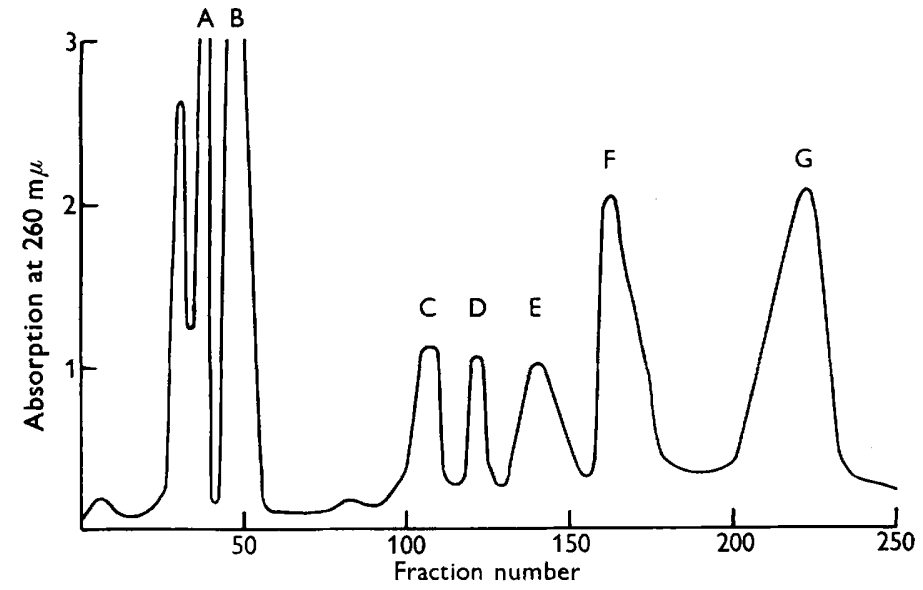

Fig. 1. Diagram of the elution of nucleotides of Lactobacillus arabinosus from Dowex-1. The lettering of the peaks refers to Table 1. 
The nucleotides found in the organisms in this survey are summarized in Table 1. Elution was discontinued after the strength of the eluant had almost reached $4 \mathrm{~N}$-formic acid, by which time adenosine diphosphate had been displaced from the column. As will be seen from Table 1, the cytidine diphosphate derivatives were limited in their distribution. CDPribitol and CDPglycerol were found in Lactobacillus arabinosus and L. plantarum. However, $\boldsymbol{L}$. bulgaricus contained only CDPglycerol, while Bacillus subtilis contained only CDPribitol. In $B$. subtilis the amount of CDPribitol found, relative to the AMP peak, was only about a fifth of that in $L$. arabinosus or $L$. plantarum. The only other organism in which these cytidine compounds were detected was Staphylococcus aureus under conditions of penicillin stasis. A small peak possessing a cytidine spectrum emerged from the column in the position normally occupied by CDPribitol, but the amounts present were too small for an investigation by paper chromatography.

\section{DISCUSSION}

It is now known that there are marked differences in the chemical composition of the cell walls of Gram-positive and Gram-negative bacteria with respect to lipid, amino acid and carbohydrate content (Salton, 1953; Cummins \& Harris, 1956; Work 1957). Mitchell \& Moyle (1954) determined the phosphoruscontaining compounds of a number of Gram-positive and Gram-negative organisms after lipid extraction, and concluded that the Gram-positive organisms contained a phosphoric ester fraction (XSP) which was absent from the Gram-negative organisms. They had previously found that in Staphylococcus aureus (Micrococcus pyogenes) at least part of this XSP appeared to be a polyglycerophosphate compound. Jones, Ritzvi \& Stacey (1958), using a more detailed method of analysis, presented evidence that this non-nucleotide phosphorus fraction occurred in preparations made from whole organisms of both Gram-positive and Gram-negative organisms. They identified two components from the hydrolysate of the XSP fraction from Klebsiella pneumoniae as glycerophosphate and inositol phosphate. It should be noted, however, that the analyses of Jones et al. (1958) show no correlation between the Gramstaining reaction and the content of ribonucleic acid or the ratio of ribonucleic acid and deoxyribonucleic acid.

The discovery that ribitol phosphate is present in a combined form in large amounts in the cell walls of Lactobacillus arabinosus, Bacillus subtilis and Staphylococcus aureus (Baddiley, Buchanan \& Carss, 1958), provided an explanation for some part of the XSP fraction. Further studies have shown that the ribitol polymer from $L$. arabinosus also contains glucose and alanine (Armstrong, Baddiley, Buchanan \& Carss, 1958). No glycerol polymer was found in the cell-wall fraction but it was suggested that it might be a component of the cytoplasmic membrane. It is only possible to distinguish clearly between the cell wall and the cytoplasmic membrane in Gram-positive bacteria where the cell wall can be stripped off by agents such as lysozyme, leaving protoplasts. Some information on the composition of the cytoplasmic membrane 


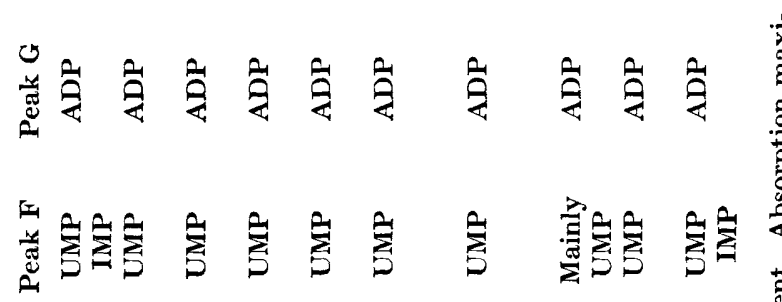



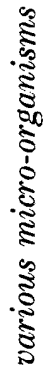

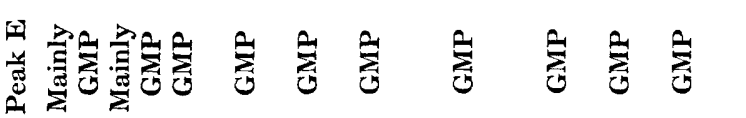

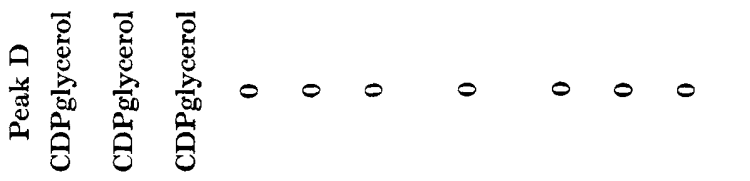

㐘

离

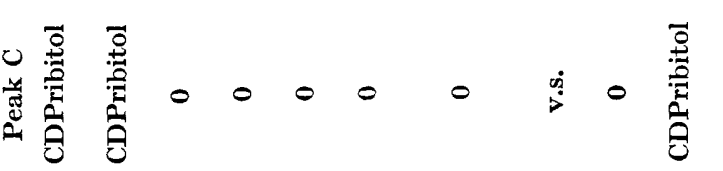

窟

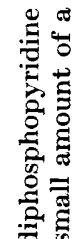

学宫

हूँ

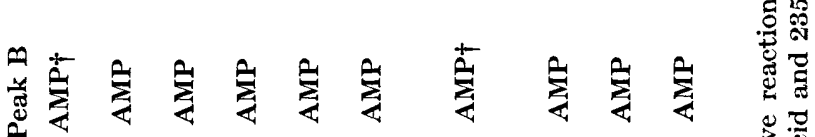

躍

हों

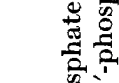

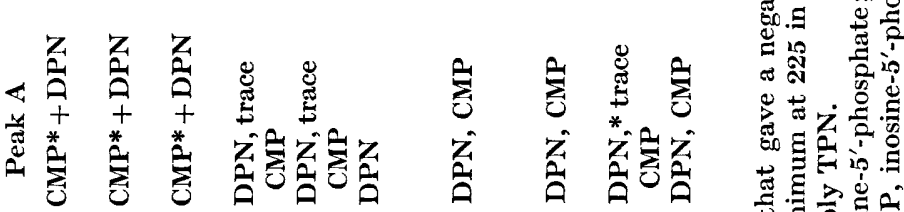

:

$\stackrel{-1}{\frac{0}{0}}$

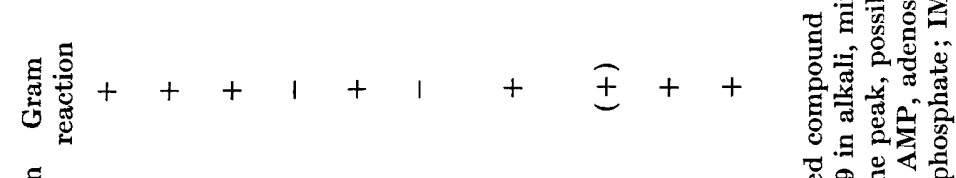

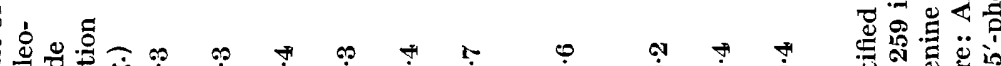

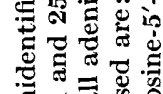

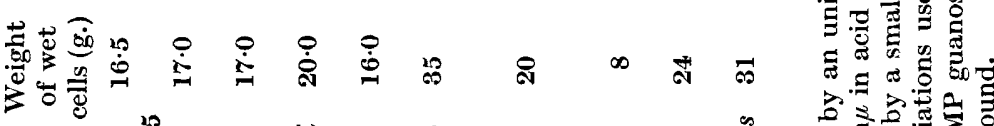

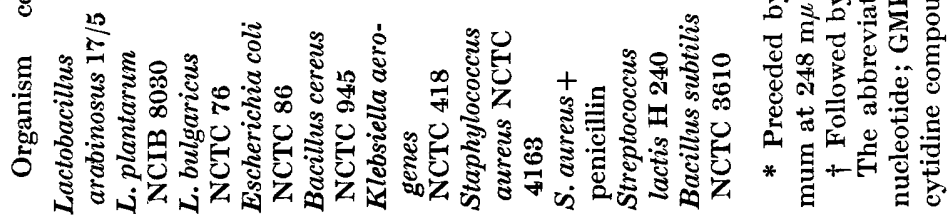


of a Gram-positive organism is given by Weibull (1957) who examined the lipids of a lysozyme-sensitive bacillus and found that $65 \%$ of the total lipids appeared to be located in the cytoskeletons remaining after the cells had been lysed with lysozyme. The lipids from this fraction appeared to consist of a mixture of neutral fats, fatty acids and phosphatidic acids. Gilby, Few \& McQuillen (1958) examined the chemical composition of the protoplast membrane of Micrococcus lysodeikticus and found that the lipid component had a similar absence of nitrogen-containing compounds and appeared to consist of approximately $80 \%$ of complex phosphatidic acids. The resistance to hydrolysis by methanolic potassium hydroxide suggested that these were not simple phosphatidic acids. The lipid fraction contained about $73 \%$ of the phosphorus present in the intact membrane, thus leaving $27 \%$ to be accounted for in the non-lipid fraction.

If a ribitol phosphate polymer is a normal cell-wall component, and a glycerophosphate polymer a normal cytoplasmic membrane component, it would seem likely that cytidine diphosphate ribitol and cytidine diphosphate glycerol are involved in their biosynthesis. If this were so, it might have been expected that the cytidine diphosphate derivatives would have been of more general distribution than this survey suggests. Failure to demonstrate these compounds may simply mean that their normal concentration within the cell is too low for detection by these methods. The experiment in which Staphylococcus aureus was treated with penicillin showed that a small amount of cytidine compound accumulated under conditions similar to those in which Park (1952) found accumulation of the uridine nucleotides which are thought to be cell-wall precursors (Park \& Strominger, 1957). If the glycerol and ribitol polymers are found to be of widespread occurrence in bacteria, then the exceptional organisms may be those which accumulate the cytidine nucleotides under normal growth conditions. Of the two Bacillus species examined, Bacillus subtilis, which has been shown by Baddiley et al. (1958) to contain a ribitol phosphate polymer in the cell wall, was found by us to contain considerable amounts of cytidine diphosphate ribitol. This was not found in the extracts of $B$. cereus and it would be interesting to know whether the cell wall of this species contains a ribitol phosphate polymer.

The distribution of the cytidine diphosphates among the lactobacilli examined fits in well with current ideas on their classification. It is not surprising that Lactobacillus plantarum NCIB 8030 should resemble $L$. arabinosus 17/5. Briggs (1953) classed L. arabinosus with $L$. plantarum and L. casei in group VI of her classification of lactobacilli based on physiological tests. Wheater $(\mathbf{1 9 5 5} b)$ also included L. arabinosus with L. plantarum. Sharpe (1955) in a serological study of lactobacilli found that four strains described as L. arabinosus fell into the $L$. plantarum serological group. Lactobacillus bulgaricus on the other hand can be distinguished from L. arabinosus and L. plantarum by physiological tests (Briggs, 1953, Wheater, 1955a), and serological reactions (Sharpe, 1955). Since only cytidine diphosphate glycerol, and not cytidine diphosphate ribitol, was found in the extracts from L. bulgaricus it would be interesting to know whether or not the ribitol polymer will be found 
in the cell wall. The species distribution of these polyol phosphate polymers in bacterial structures may turn out to be fairly wide, if they can be equated with all or part of the XSP fractions described by Mitchell \& Moyle (1954) and Jones et al. (1958). If this should prove to be the case, it would be interesting to know the factors which cause the accumulation of relatively large amounts of the cytidine nucleotides, which may be precursors, in the species we have described. In considering the possible functions of CDPribitol and CDPglycerol, it is worth noting that in the biosynthetic reactions of both the other CDP compounds, CDPcholine and CDPethanolamine, cleavage of the pyrophosphate link occurs. Thus if these compounds are represented by the general formula CDPX, then it is PX that is transferred. It might be argued that in the case of the polyol derivatives of cytidine diphosphate, one would expect to find that it was the polyol phosphate that was incorporated as a unit in any polymerization reactions. This is in contrast to the reactions of the sugar derivatives of uridine diphosphate (UDP). In the transferase systems in which these take part, the sugar is transferred without a phosphate ester group and UDP is formed.

The assistance of Helen Green is gratefully acknowledged in the later stages of this work.

\section{REFERENCES}

Armstrong, J. J., Badpilesy, J., Buchanan, J. G. \& Carss, B. (1958). Nucleotides and the bacterial cell wall. Nature, Lond. 181, 1692.

Baddiley, J., Buchanan, J. G. \& Carss, B. (1956). The configuration of the ribitol phosphate residue in cytidine diphosphate rtbitol. J. chem. Soc. p. 1869.

Baddiley, J., Buchanan, J. G. \& Carss, B. (1958). The presence of ribitol phosphate in bacterial cell walls. Biochim. biophys. Acta, 27, 220.

Baddiley, J., Buchanan, J. G., Carss, B. \& Mathias, A. P. (1956). Cytidine diphosphate ribitol from Lactobacillus arabinosus. J. chem. Soc. p. 4583.

Baddiley, J., Buchanan, J. G., Carss, B., Mathias, A. P. \& Sanderson, A. R. (1956). The isolation of CDPglycerol, CDPribitol and Manitol 1-Phosphate from Lactobacillus arabinosus. Biochem. J. 64, 599.

Baddiley, J., Buchanan, J. G., Handschumacher, R. E. \& Prescott, J. F. (1956). Chemical studies in the biosynthesis of purine nucleotides. Part I. The preparation of $N$-glycylglycosylamines. J. chem. Soc. p. 2818.

Baddiley, J., Buchanan, J. G., Mathias, A. P. \& Sanderson, A. R. (1956). Cytidine diphosphate glycerol. J. chem. Soc. p. 4186.

Baddiley, J. \& Mathias, A. P. (1954). Cytidine nucleotides. Part I. Isolation from Lactobacillus arabinosus. J. chem. Soc. p. 2723.

Baer, E. \& Fischer, H. O. L. (1939). Studies on Acetone-Glyceraldehyde. VI. Synthesis of the biological $\mathrm{L}(-) \propto$-glycerophosphoric acid. J. biol. Chem. 128, 491.

Briggs, M. (1953). The classification of lactobacilli by means of physiological tests. J. gen. Microbiol. 9, 234.

Caputto, R., Leloir, L. F., Cardini, C. E. \& Paladini, A. C. (1950). Isolation of the coenzyme of the galactose phosphate-glucose phosphate transformation. J. biol. Chem. 184, 333.

Cummins, C. S. \& Harris, H. (1956). The chemical composition of the cell wall in some Gram-positive bacteria and its possible value as a taxonomic character. J. gen. Microbiol. 14, 583.

Drake, B. (1955). Contributions to the theory of gradient elution analysis. Ark. Kemi, 8, 1. 
Gilby, A. R., Few, A. V. \& McQulllen, K. (1958). The chemical composition of the protoplast membrane of Micrococcus lysodeikticus. Biochim. biophys. Acta, $29,21$.

Hanes, C. S. \& Isherwood, F. A. (1949). Separation of the phosphoric esters on the filter paper chromatogram. Nature, Lond. 164, 1107.

Hurlbert, R. B., Schmitz, H., Brumm, A. F. \& Potter, V. R. (1954). Nucleotide metabolism. II. Chromatographic separation of acid soluble nucleotides. $J$. biol. Chem. 209, 23.

Jones, A. S., Ritzvi, S. B. H. \& Stacey, M. (1958). The phosphorus-containing compounds of Gram-positive and Gram-negative organisms in relation to the Gram staining reaction. J. gen. Microbiol. 18, 597.

Kennedy, E. P. \& Weiss, S. B. (1956). The function of cytidine coenzymes in the biosynthesis of phospholipids. J. biol. Chem. 222, 193.

KodiceK, E. \& ReDDI, K. K. (1951). Paper chromatography of nicotinic acid derivatives. Nature, Lond. 168, 475.

Magasanik, B., Vischer, E., Doniger, R., Elson, D. \& Chargaff, E. (1950). The separation and estimation of ribonucleotides in minute quantities. $J$. biol. Chem. 186, 37.

Marshak, A. \& Vogel, H. J. (1951). Microdetermination of purines and pyrimidines in biological materials. J. biol. Chem. 189, 597 .

Mrtchell, P. \& Moyle, J. (1951 $a$ ). Isolation of hydrolytic products of a glycerophospho-compound from Micrococcus pyogenes. J. gen. Microbiol. 5, 966.

Mrtchell, P. \& Moyle, J. (1951b). The glycerophospho-protein complex envelope of Micrococcus pyogenes. J. gen. Microbiol. 5, 981.

Mitchell, P. \& Moyle, J. (1954). The Gram reaction and cell composition: Nucleic acids and other phosphate fractions. J. gen. Microbiol. 10, 533.

Paladini, A. C. \& Lelorr, L. F. (1952). Studies on uridine-diphosphate-glucose. Biochem. J. 51, 426.

Park, J. T. (1952). Uridine-5'-pyrophosphate derivatives. Isolation from Staphylococcus aureus. J. biol. Chem. 194, 877.

Park, J. T. \& Strominger, J. L. (1957). Mode of action of penicillin. Science, 125, 99.

Salton, M. R. J. (1953). The composition of the cell walls of some Gram positive and Gram-negative bacteria. Biochim. biophys. Acta, 10, 512.

SharPe, M. E. (1955). A serological classification of lactobacilli. J. gen. Microbiol. $12,107$.

Weibuld, C. (1957). The lipids of a lysozyme sensitive Bacillus species. (Bacillus 'M'). Acta chem. Scand. 11, 881.

Wheater, D. M. $(1955 a)$. The characteristics of Lactobacillus acidophilus and Lactobacillus bulgaricus. J. gen. Microbiol. 12, 123.

Wheater, D. M. $(\mathbf{1 9 5 5} b)$. The characteristics of Lactobacillus plantarum, L. helveticus and L. casei. J. gen. Microbiol. $12,133$.

Work, E. (1957). Biochemistry of the bacterial cell wall. Nature, Lond. 179, 841.

WyatT, G. R. (1951). The purine and pyrimidine composition of deoxypentose nucleic acids. Biochem. J. 48, 584. 